\title{
Innovative and Effective Methodology for Determining PE Learning Outcomes in Bulgarian School
}

\author{
Yordanka St. Dimitorva* (a)
}

(a) Secondary school “Geo Milev”, 124a Mladost, Varna(Bulgaria), iordanka_d@gbg.bg

\begin{abstract}
The problem of physical education and sports, as an integral part of the learning process in the Bulgarian school, which has a significant impact on the overall formation of the student's personality, is extremely relevant and significant, especially in terms of its effective organization and reporting. As a purposeful and organized process, physical education and sports are normatively conditioned, that is it is planned, organized and reported according to established state rules and norms - such as mandatory and standardized tests of the Ministry of Education and Science (MES), for example, which in their entirety place emphasis on the physical capacity of students. This circumstance reflects on the goals and objectives set in the curricula for the subject of physical education in the Bulgarian school, which do not affect the age and individual characteristics of students and do not refer to clear and precise criteria for reporting the results.

All this creates conditions for directing the efforts of physical education teachers to the implementation of activities that do not fully form the physical culture of students, on the one hand, and on the other, create conditions for its biased and incorrect reporting and evaluation.

The purpose of this article is to prove the relevance of the problem of the need for more effective reporting of the results of physical education and sports in the Bulgarian school through the application of innovative methods included in a developed and tested author's theoretical model.
\end{abstract}

Keywords: physical education and sport, innovative methods, effective methods, theoretical model, physical capacity, physical development.

\section{(C) 2020 Yordanka St. Dimitrova}

This is an open access article distributed under the terms of the Creative Commons Attribution License (CC BY 4.0), which permits unrestricted use, distribution, and reproduction in any medium, provided the original author and source are credited.

Published by Kazan federal university and peer-reviewed under responsibility of IFTE-2020 (VI International Forum on Teacher Education)

* Corresponding author. E-mail: iordanka_d@gbg.bg 


\section{Introduction}

Physical education appears as a clear need for the development of a physically strong, beautiful and resilient body and a spirit adequate to it. In most cases, it is perceived as a dynamic and complex system, which inevitably bears the marks of the era, the peculiarities of society and the nation, in which it develops and is implemented in practice in the form of a purposeful process of education and upbringing as the purpose of formation and development of the human personality (Filev, 2005).

Pedagogical science proves in an indisputable way that physical education is a structural component of the content of education. This gives grounds to claim that his pedagogical model includes the purposeful, systematic and specially organized interaction between the main subjects of the educational process and of these subjects with the surrounding environment (physical, social and organizational).

In the conditions of the educational institution school, it should be emphasized the fact that education in physical education and sports has an organized and purposeful character, i.e. physical education is normatively conditioned. It's closely linked to specific laws and regulations, which in their entirety place emphasis on the physical development and physical capacity of the student's personality.

\section{Literature Review}

In the conditions of the Bulgarian school, the training in physical education and sports for each stage is determined by the content of the programs of the MES, which are mandatory (Law on Public Education, 1991; Health Act, 2004). In terms of content, the programs include topics and activities in compliance with the state general education requirements for the process of education in physical education and sports, as well as with the specific features of the students in the respective class and with the conditions available to the respective school (Ministry of Health, 2003; National Strategy for Development of Physical Education and Sports in the Republic of Bulgaria 2012-2022, 2011). In all programs, the emphasis is placed on the formation and development of qualities related mainly to the physical capacity of the student, and not on the level of his upbringing and physical culture in general (Law on the level of education, the general education minimum and the curriculum, 1999; Law on Physical Education and Sports, 1996). That is, the programs focus on the formation and development of physical skills and qualities in students, but not on the formation of knowledge and values towards health and a healthy lifestyle. At this stage, clear criteria and indicators have not been developed for: assessment of the level of mastery of sports terminology; about the essence of physical education and physical culture; about the connection and dependence of physical education with human health (mental and physical); for the structure, content and impact on the person of the active physical activities; for the level of formation of personal skills and qualities (physical: strength, 
agility, speed, etc. and mental: communication, empathy, will, skills for decision making and dealing with difficult situations, etc.; for the level of formation and development the motivation of the student for active participation in the classes of physical education and sports. All this draws attention to the application of tests and standards of the Ministry of Health ( $\mathrm{MH})$, which complement and expand the range of indicators to take into account the physical capacity and physical development of students (Damyanova \& HristovaKotseva, 2007).

The development and application of such criteria and indicators would also support the learning process for objective determination of the level of formation of the physical culture of the students, in the class of physical education and sports (Piko, 2007).

\section{Purpose and objectives of the study}

Aim of the research: To prove the effectiveness of innovative methods for determining the results of the training in physical education and sports in the Bulgarian school, included in the author's theoretical model, through experimental research in the junior high school stage. The object of the research is the effectiveness of innovative methods for determining the results of the training in physical education and sports in the Bulgarian school, included in the author's theoretical model. The subject of the research is highlighting the effectiveness of innovative methods for determining the results of physical education and sports training in the Bulgarian school, included in the author's theoretical model, through experimental research in the junior high school stage.

\section{Methodology}

A total of 292 students (146 boys and 146 girls) from four primary schools participated in the research experiment. The students participating in the experiment are aged 12 to 14 years and are distributed according to the following indicators, as follows: number, gender; classes and school.

All schools are located in Varna. They are "Chernorizets Hrabar","Zahari Stoyanov", "Petko Rachev Slaveykov" and "Ivan Vazov".

The sample of students is differentiated into the following subgroups:

1) Two experimental groups from "Chernorizets Hrabar" Primary School:

- 55 students in the period of study from fifth to seventh grade, of which 28 boys and 27 girls;

- 59 students in the period of study from fifth to sixth inclusive 29 boys and 30 girls; 
2) Two control groups from the other schools included in the research:

- 80 same-age students in grade 7 of which 35 boys and 45 girls;

- 98 students of different ages, of which 54 boys and 44 girls.

\section{Stages of the experiment}

The experimental work covers a five-year period (2009 to 2014), in which four main stages stand out:

First stage (2009/2010) - establishing the level of formation of physical capacity and physical development of students in the first experimental group, by applying standard tests of MES and tests of MH, through an author's model of a test battery.

Second stage (2010/2012) - approbation of the author's theoretical model and complex program for students from the first and second experimental groups of "Chernorizets Hrabar" Primary School - Varna.

Third stage (2012/2013) - reporting and analysis of the results of the approbation of the theoretical model and the complex program, in the two experimental groups; processing, summarizing and analyzing the results of the survey to establish the attitude of students from both control groups to physical activity and healthy lifestyle.

Fourth stage (2013/2014) - preparation of a comparative analysis of the results of the study of the process of formation of physical culture of students and their value attitude to physical activity and healthy lifestyle of students from both experimental and two control groups.

Research methods: survey method; targeted monitoring; testing and pedagogical experiment. Methods for processing the research results: variation analysis; Student's comparative $\mathrm{t}$ criterion; sigma deviation method; method of indices - body mass index (Body Mass Index) and Kettle index.

\section{Results}

The results of the experimental study are presented in the logical sequence of the individual stages of its implementation.

The first stage is characterized by conducting diagnostic procedures to establish the level of formation of the qualities that determine the state of physical capacity and physical development of students from the main experimental group, studying in four primary schools in Varna. At the beginning of this stage, students' achievements are reported on the basis of mandatory indicators and criteria included in standardized MES tests. 
In connection with the formed author's idea and the purpose of the experimental research, additional tests, developed and approved by the Ministry of Health (MH). By combining the mandatory tests of the MES and standards of the $\mathrm{MH}$, an opportunity is sought for a fuller account of the level of physical capacity, in connection with and dependent the physical development on the students. This author's thesis leads to the development and experimental application of a "test battery" which includes a total of 16 indicators (9 for determining the physical capacity and 7 for determining the physical development of students). The application of the test battery and reporting of its results is carried out in the presence of the medical team in the respective school, which includes a doctor and a nurse.

The analysis of the measurement data of the students from the main experimental group on mandatory and additional indicators included in the test battery gives grounds to conclude that they are at a relatively high level.

From this point of view, in themselves, the results obtained can't serve as the only basis for objectively outlining the level of formation of the general physical culture of students, as it is not expressed only by their physical capacity and physical development. At the same time, however, it should be taken into account that the very normative base, on the basis of which the level of physical culture of students in our country is determined, does not provide for compliance of the process of physical education with individual characteristics, the needs and interests of the students, therefore it does not provide opportunities for choice of activities.

All this gives grounds to apply, in a real educational environment, the developed author's theoretical model, with the help of which to carry out an experimental-transforming activity in terms of content, organization and conduct of the training process in physical education and sports.

The application of the model took place in the form of a complex of experimental - transformational activities, within the second main stage of the research, in the primary school "Chernorizets Hrabar". Here we should pay attention to the fact that due to the innovative nature of the model, most of the transformational activities in it are not regulated in the main regulations for organizing and conducting training in physical education and sports in Bulgarian schools and are not implemented in full. at the present stage, but are directly related to the solution of the researched problem, namely:

- systematic acquaintance of students with theoretical aspects, as well as with basic concepts, rules and norms of behavior in the field of physical education; 
- systematic discussion and analysis with students of different behavioral situations in order to transform the information they receive into views and beliefs (formation of consciousness and attitude / position);

- developing and conducting individual activities with students

- developing and applying incentives for forming a positive attitude and motivating active participation in physical education classes

- application of various diagnostic procedures in order to timely identify changes in the development of the student's personality, incl. and their timely correction (formation of skills for self-control and self-assessment), etc.

For the more effective implementation of the complex of activities, within the powers of the physical education teacher, a Sample complex program for organization and development of physical education and sports in primary school is developed and implemented, as an effective tool for forming knowledge, skills and habits in students.

The adoption of this approach in the application of the model is subject to the belief that in this way it provides a better opportunity to clearly highlight the desired and desired change in the legislation governing the training in physical education and sports, and in its content, structural and organizational aspects.

Particular attention is paid to the activities for formulating goals and objectives (such as desired and prepredicted end results) in the cognitive, motor and behavioral areas. The searches are also focused on the selection of a variety of activities for the formation of knowledge, skills and habits, including and for a healthy lifestyle, which for the most part do not appear as mandatory in the normative documents of the MES. Attention is also paid to the thematic diversity of the educational content, as well as to the selection of modern tools and approaches, with the help of which to achieve greater efficiency in the implementation of the set goals and objectives. In the process of approbation of the model, attention is paid to the selection and application of a variety of methods for training and education, including explanation, demonstration, instruction, observation, discussion, discussion, small group work (team), exercise, individual work, and others.

The third stage of the experimental research is dedicated to the measurement and analysis of the results of the approbation of the theoretical model. Within the stage, the level of physical capacity and physical development of the students from the main experimental group of "Chernorizets Hrabar" Primary School Varna is established through the application of the author's development of a test battery, as well as other 
research methods, such as survey and purposeful observation. In order to more clearly outline the variety of qualities that are formed in the process of approbation of the model, the specially developed criteria and indicators are applied. The criteria include the level of acquisition of knowledge about physical education, sports and healthy lifestyle; the level of formation of qualities and the level of formation of a healthy lifestyle. The condition of all criteria is measured using a total of three indicators of high, medium and low degree of formation of motor skills.

The level of formation of the motor qualities of the students is determined by the set of qualities included in the test battery.

In order to get a more complete idea about the development of the capacity of the students from the main experimental group, after the experimental-transforming activity, a model for the development of the physical capacity is built (Figure 1). The estimates for each of the applied tests converted into points (i.e. in dimensionless quantities), are accepted as the main basis for the construction of the model. The maximum number of points that each student can receive is 30 .

It should be noted here that by applying this approach the achievements of the students from the experimental group can be compared with different units of measurement (sec., sm, kg., etc.), and by visualizing the model it is easier to determine both the changes in their individual achievements and in the achievements of the whole class.

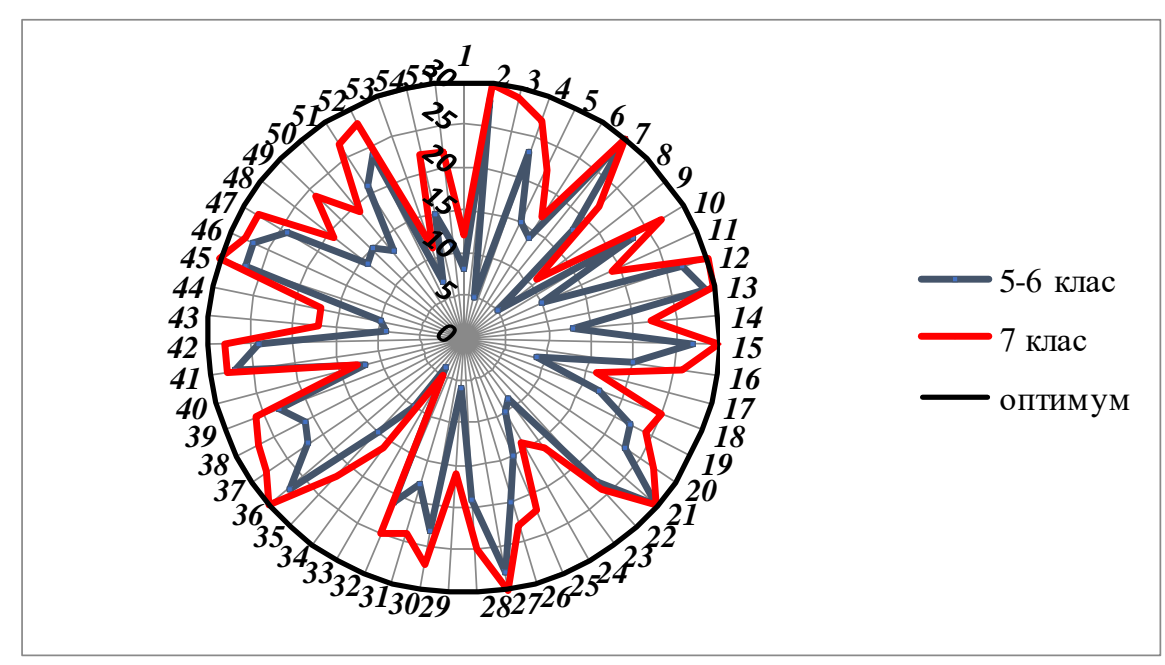

Figure 1. Model for the development of the physical capacity of the students from the experimental group (5-7 grade) from "Chernorizets Hrabar" Primary School in Varna 
However, the acceptance of the average values of the achievements from the previous classes for the main experimental group (fifth and sixth class) as control values do not give the right to draw serious conclusions before checking whether these differences are significant or not. To establish this, as indicated in the Study Methodology, Student's t-test (for dependent and independent samples) is applied when comparing the quantitative variables with the normal distribution.

After establishing the significance of the differences between the average levels of the studied traits with the help of the sigma method (T-assessments) it becomes possible to build optimization models of physical development and physical capacity of girls (See Figure 2.) and boys (See figure No 3) from "Chernorizets Hrabar "Primary School - Varna, included in the experimental group.

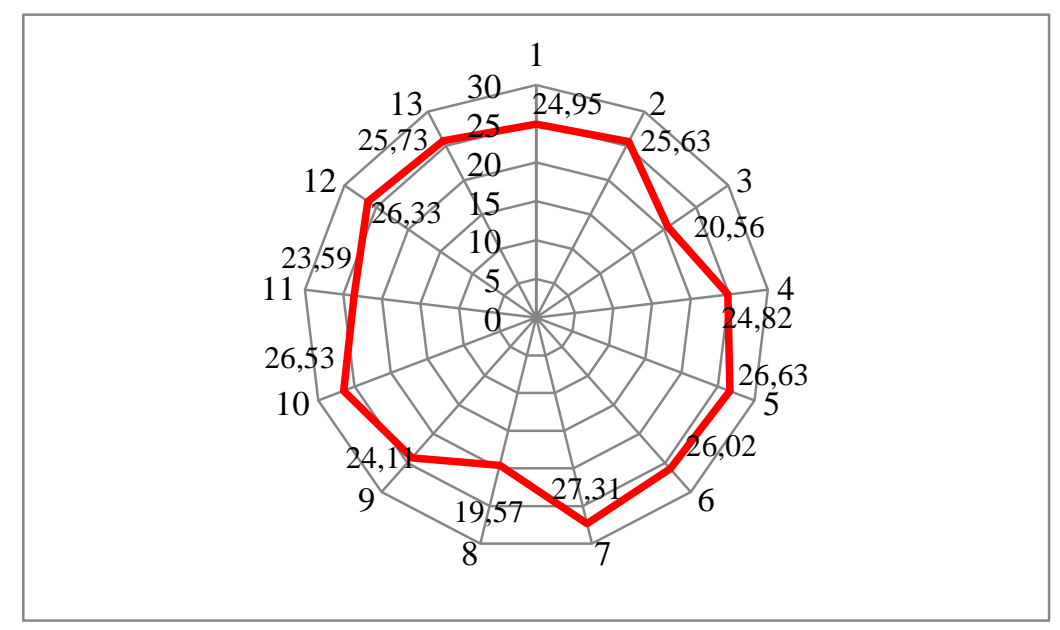

Figure 2. Optimization model of the physical development and physical capacity of the girls from “Chernorizets Hrabar" Primary School

In order to highlight even better the efficiency of the application of the theoretical model, ie. to establish whether the qualities formed in the students, through the training in physical education and sports are perceived and realized by them as values, a survey method is applied. In connection with the requirements for its correct application, a total of three questionnaires are developed: to establish the level of development of physical and mental qualities in students; to establish the attitude and motivation of students to physical activity and a healthy lifestyle and to establish the attitude and motivation of students to healthy eating.

The analysis of the results obtained from the application of the questionnaire method shows that the students who participated in the experimental-transformational activity in the application of the theoretical 
model have the necessary skills to identify the qualities that they believe are formed in physical education and sports. of these qualities moves in close values, and the qualities that are indicated as necessary to the modern personality largely overlap with those that students believe they possess personally. The surveyed students have a much clearer positive attitude towards the physical and mental qualities that are formed in them through physical education and sports classes, as well as under the influence of various other factors. Along with this, the presence of prerequisites for students to form a value attitude towards active exercise and sports games is confirmed. The presence of a clearly expressed value orientation and motivation in them for systematic physical activity and a healthy lifestyle stands out. The analysis shows that for the most part, students distinguish between healthy and unhealthy lifestyles (hygiene requirements, diet and exercise, food and drink choices, etc.).

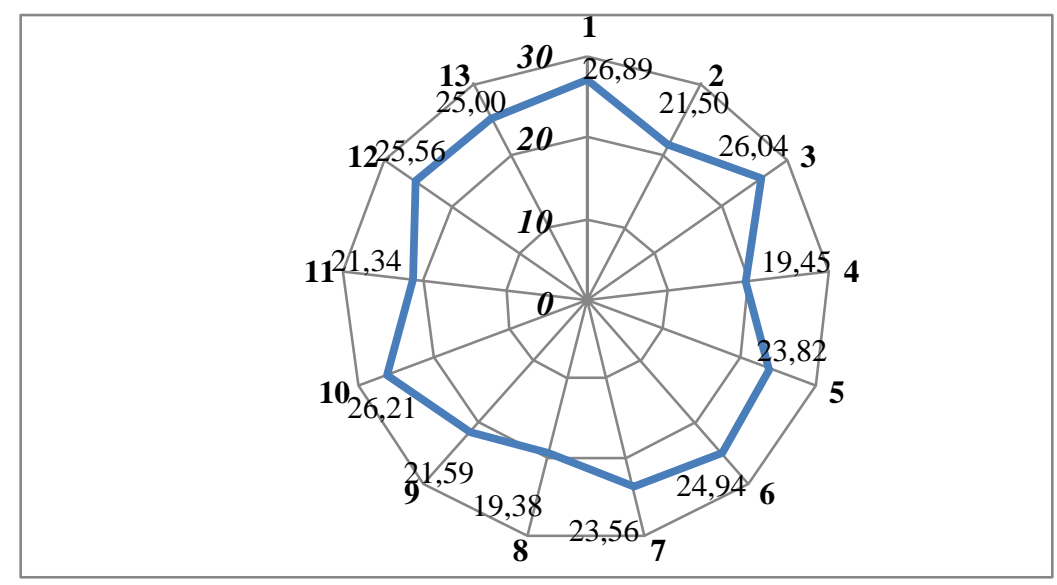

Figure 3. Optimization model of the physical development and the physical capacity of the boys from "Chernorizets Hrabar" Primary School

For the overall evaluation of the results of the experimental - transforming activities, which accompany the approbation of the theoretical model, specially developed by the author criteria and indicators are applied.

Based on the assessment, it is evident that the theoretical model, in its entirety, has a positive effect on increasing the efficiency of physical education and sports classes. It contributes both to the growth of the main qualitative indicator for establishing the physical culture of students: physical capacity, and to their general physical development, through the application of tests of the MH. The inclusion of new content, organizational and activity moments in the process of training in physical education and sports has a beneficial effect on the awareness of the importance of physical activity and healthy lifestyle as vital factors for the overall formation and development of the students of the experimental group. This 
circumstance has a positive impact on increasing their internal motivation for active participation in the class, as well as for their inclusion in extracurricular and extracurricular sports activities.

On the basis of the conducted survey, it is established that a large part of the qualities formed in the students in the class of physical education and sports are perceived by them as values (physical development, development of motor skills and habits; basic mental qualities.

The fourth stage is dedicated to the preparation of a comparative analysis of the results of the survey of the main experimental group with the results of the one-age control group and the results of the second experimental group with the results of the second control group

The first analysis shows that the students who participate in the experimental-transformational activity, i.e. in the process of applying the theoretical model have the necessary skills to identify the qualities that they believe are formed in the class of physical education and sports, as the gradation of these qualities moves in close values, to a much higher degree than students included in the same age control group. The students from the experimental group have a much clearer positive attitude to the physical and mental qualities that are formed in them through physical education and sports, as well as under the influence of various other factors than their peers from the control group. Along with this, the presence of many more prerequisites in the students from the main experimental group for the formation of a value attitude to active activities with physical exercises and sports games than in those of the control group is established. The former also has a much clearer value orientation and motivation for systematic physical activity and a healthy lifestyle than the latter. It is clear from the analysis that for the most part, the students who participated in the experimental-transformational activities included in the theoretical model make a much more accurate and reasoned distinction between healthy and unhealthy lifestyles (hygiene, nutrition and exercises) than students who didn't participate in the approbation.

To strengthen the belief that the included innovative methods in the developed and tested in practice theoretical model increase the effectiveness of reporting the results of physical education and sports in the Bulgarian school came the idea of its application in physical education and sports classes of the new class of students, accepted for training in the "Chernorizets Hrabar" Primary School. To more clearly distinguish the group from all others included in the study, it is defined as the second experimental. The model is applied in the first year of their training, i.e. in the fifth grade. The results are reported and analyzed in the next school year, when students move to a higher (sixth) grade, based on the application of the questionnaire method, using the same questionnaires used in the main experimental group and the one-age control group. 
The surveys are also conducted with the sixth-grade students, from the so-called second control group (of different ages), which includes students from "Z. Stoyanov", "P.R.Slaveykov" and "Iv. Vazov" primary schools in Varna.

The comparative analysis of the results of this study again shows a clear difference in the opinion of the students from the two groups - on all indicators. While the students from the second experimental group demonstrate knowledge related to the precise determination of the qualities (mental and physical) that are formed in the physical education and sports class, those from the second control group have difficulties.

This circumstance also affects the answers of the students from both groups in connection with determining the degree of possession of these qualities. Similar to the comparative analysis in the main experimental group and the first control group, the presence of more prerequisites for the formation of a value attitude towards active exercise and sports games in the students of the second experimental group than in the students of the second control group. While the former has a much clearer value orientation and motivation for systematic physical activity and a healthy lifestyle, the latter lack it. The analysis shows that students who participated in the experimental transformation activities included in the theoretical model, for the most part, distinguish between healthy and unhealthy lifestyles (hygiene, diet and exercise, food and beverage choices). etc.), while the students from the second control group cannot make such a difference.

Based on the analysis of the results obtained by applying the method of comparative analysis and the method of targeted observation, the belief is confirmed that the training in physical education and sports in students and the second experimental group contributes to the formation of lasting motor skills (and in high degree), as well as moral, aesthetic and labor (respectively in medium and high degree). This statement is also conditioned by the established sustainable behavioral manifestations, such as discipline, organization, empathy, responsibility, awareness, mutual assistance, cooperation, humanity, initiative, maintaining the good appearance and personal hygiene, active and conscious participation in the arrangement, protection and preservation of sports equipment and facilities.

The comparison of the results obtained after the implementation of the experimental-transforming activity, accompanying the approbation of the innovative methods included in the author's theoretical model in the classes of physical education and sports with the participation of two experimental groups in the school of the other schools included in the study and their analysis, gives grounds to claim that the model works in the conditions of the modern school and increases the efficiency of physical education and sports in the presence of certain conditions. 


\section{Discussions}

Based on the results of the conducted experimental and transformational research, the following discussion questions can be highlighted:

- The need to rethink the importance of education in physical education and sports, as a means of complete formation of the student's personality, by all social factors.

- Need for revision of the normative base, determining the school physical education and sports and its compliance with the individual and age peculiarities of the students and the parameters of the existing sports base.

- Need for the unification of the standards and tests of the Ministry of Education and Science (MES) and the Ministry of Health $(\mathrm{MH})$ with regard to the effective formation and full consideration of the physical capacity and physical development of students.

- Need to develop a unified methodology for training and evaluation of the results of physical education and sports of students in the school education system.

- Need for permanent training of teachers of physical education and sports as key actors in the training process for effective planning, organizing, conducting, diagnosing and evaluating this process.

- Need for systematic orientation of students to active physical activity and a healthy lifestyle, through the application of various methods and means for stimulation and motivation, incl. and by the example of adults.

- Need for research and wide discussion of innovative conceptual and theoretical-practical developments of teachers of physical education and sports, in order to apply effective practices.

\section{Conclusion}

The conclusions from the conducted experimental research give grounds to assume that the problem of developing and applying in educational practice innovative methods for reporting the results of physical education and sports in the Bulgarian school is extremely relevant, personally and socially significant. As a public task set for implementation by the school institution, physical education is defined as a system (as a single process) with rationally organized components, which affects the overall formation and development 
of the student's personality (Stanimirova, 2007). In this aspect, physical education should be considered as a set of physical and spiritual values aimed at the physical improvement and harmonious development of the individual, to the construction of his physical culture.

The results of the conducted experimental research clearly prove that at this stage in the Bulgarian school, the level of physical culture of students is determined primarily by normative indicators approved by the Ministry of Education and Science. The experimentally developed and practically applied test battery, which includes both the regulated tests of the Ministry of Education and Science and additional (optional) tests of the Ministry of Health, not only expands the spectrum of reporting the set of qualities that characterize more fully and comprehensively the physical culture of students and outlines the problem areas in the planning, organization and conduct of physical education and sports classes, namely the consideration of the individual and age characteristics of students, on the one hand, and on the other existing material conditions. An attempt to overcome the identified shortcomings was made by carrying out a set of transformational activities, included in the content developed for the purposes of experimental research author's theoretical model and exemplary comprehensive program for organizing and developing physical education and sports in primary school as an effective tool for the overall formation of students (Rusev \& Ivanov, 2007).

The application of the criteria, indicators and indicators specially developed for the purposes of the research contribute to the more objective assessment of the results of the transformation activities, which accompany the approbation of the theoretical model. Based on the prepared assessment, it is concluded that the application of the theoretical model has a generally positive effect on increasing the efficiency of physical education and sports classes. It contributes both to the growth of the main qualitative indicator for establishing the physical culture of students: physical capacity, and to their general physical development, through the inclusion of tests within the test battery and the MH, together with those of the MES. The renewal of the content, organizational and activity moments in the process of training in physical education and sports also contribute to the awareness of the importance of physical activity and healthy lifestyle as vital factors for the overall formation and development of students' personalities.

There are skills for identification and objective assessment of the main factors determining the process of value formation in junior high school students, as well as skills for self-organization, self-control and selfcorrection in them. Sustainable forms of healthy behavior also stand out. 


\section{References}

Damyanova, M. R., \& Hristova-Kotseva, R. (2007). Physical education and development. Purpose and tasks. Means of physical education. Forms of work. Pedagogy. Theory of upbringing (first part). Sofia: Libra.

Filev, F. (2005). Physical education: Methods and technical means for research. Sofia: St. Klimen Ohridski.

Health Act. (2004). Retrieved from http://www.lex.bg/bg/laws/ldoc/2135489147

Law on Public Education. (1991). Retrieved from http://lex.bg/bg/laws/ldoc/2132585473

Law on the level of education, the general education minimum and the curriculum. (1999). Retrieved from https://www.mon.bg/?h=downloadFile\&fileId=37

Law on Physical Education and Sports. (1996). Retrieved from http://www.lex.bg/bg/laws/ldoc/2133881857

Ministry of Health. (2003). Ordinance № 2 on the organization and conduct of preventive examinations for persons from 0 to 18 years of age and dispensary monitoring of compulsorily insured persons. Retrieved from http://econ.bg

National Strategy for Development of Physical Education and Sports in the Republic of Bulgaria 20122022. (2011). Retrieved from http://mpes.government.bg/Documents/Documents/Strategii/Strategia_2012-2022.pdf

Piko, B. F. (2007). Self-perceived health among adolescents: the role of gender and psychosocial factors. European journal of pediatrics, 166(7), 701-708.

Rusev, R., \& Ivanov, S. (2007). Diagnostic systems in physical education. Blagoevgrad: Neofit Rilski.

Stanimirova, N. (2007). Physical and pubertal development of Bulgarian children from 0 to 18 years of age: norms and standard curves. Sofia: Author. 